\title{
A Specific Brain Structural Basis for Individual Differences in Reality Monitoring
}

\author{
Marie Buda, ${ }^{1,2}$ Alex Fornito, ${ }^{2,3}$ Zara M. Bergström, ${ }^{1,2}$ and Jon S. Simons ${ }^{1,2}$ \\ ${ }^{1}$ Department of Experimental Psychology, and ${ }^{2}$ Behavioural and Clinical Neuroscience Institute, University of Cambridge, Cambridge CB2 3EB, United \\ Kingdom, and ${ }^{3}$ Melbourne Neuropsychiatry Centre, University of Melbourne, Victoria 3010, Australia
}

Much recent interest has centered on understanding the relationship between brain structure variability and individual differences in cognition, but there has been little progress in identifying specific neuroanatomical bases of such individual differences. One cognitive ability that exhibits considerable variability in the healthy population is reality monitoring; the cognitive processes used to introspectively judge whether a memory came from an internal or external source (e.g., whether an event was imagined or actually occurred). Neuroimaging research has implicated the medial anterior prefrontal cortex (PFC) in reality monitoring, and here we sought to determine whether morphological variability in a specific anteromedial PFC brain structure, the paracingulate sulcus (PCS), might underlie performance. Fifty-three healthy volunteers were selected on the basis of MRI scans and classified into four groups according to presence or absence of the PCS in their left or right hemisphere. The group with absence of the PCS in both hemispheres showed significantly reduced reality monitoring performance and ability to introspect metacognitively about their performance when compared with other participants. Consistent with the prediction that sulcal absence might mean greater volume in the surrounding frontal gyri, voxel-based morphometry revealed a significant negative correlation between anterior PFC gray matter and reality monitoring performance. The findings provide evidence that individual differences in introspective abilities like reality monitoring may be associated with specific structural variability in the PFC.

\section{Introduction}

Remembering a previous experience often involves distinguishing information that was generated by internal cognitive functions (e.g., thought and imagination) from information that was derived from the outside world, an ability termed "reality monitoring" (Johnson and Raye, 1981). A number of neuroimaging studies have investigated the brain basis of reality monitoring processes, with an emerging consensus that medial regions of anterior prefrontal cortex (PFC) are among the brain areas involved (Mitchell and Johnson, 2009). For example, activity in this area differentiates between recollection of whether stimuli were previously seen or imagined by a participant (Kensinger and Schacter, 2006; Simons et al., 2006), as well as whether a task was previously performed by the participant or another person ( $\mathrm{Si}$ mons et al., 2008; Lagioia et al., 2011).

Although we may feel that our reality monitoring abilities are reliable, there is evidence of substantial variability in accuracy across individuals. This is most apparent in the reality monitoring

\footnotetext{
Received July 14, 2011; revised July 28, 2011; accepted July 31, 2011.

Author contributions: A.F. and J.S.S. designed research; M.B. and A.F. performed research;M.B., Z.M.B., and J.S.S. analyzed data; M.B., A.F., Z.M.B., and J.S.S. wrote the paper.

This work was supported by Biotechnology and Biological Sciences Research Council (United Kingdom) Grant BB/G014795/1 to J.S.S.; a Medical Research Council (United Kingdom) studentship to M.B.; and National Health and Medical Research Council (Australia) CJ Martin Fellowship 454797 to A.F. The work was completed within the University of Cambridge Behavioural and Clinical Neuroscience Institute, funded by a joint award from the Medical Research Council (United Kingdom) and the Wellcome Trust.

Correspondence should be addressed to Dr. Jon S. Simons, Department of Experimental Psychology, University of Cambridge, Downing Street, Cambridge CB2 3EB, UK. E-mail: jss30@cam.ac.uk.

DOI:10.1523/JNEUROSCI.3595-11.2011

Copyright $\odot 2011$ the authors $\quad 0270-6474 / 11 / 3114308-06 \$ 15.00 / 0$
}

impairments often observed in patients with clinical disorders such as schizophrenia (Keefe et al., 2002), but variability in performance is also seen in apparently healthy volunteers (Hyman and Billings, 1998). As an illustration, Figure 1 shows the performance of 146 healthy, young adult volunteers who undertook one of a number of reality monitoring tasks. As can be seen, reality monitoring accuracy varied considerably between individuals.

Currently, the relationship between brain structure variability and individual differences in cognitive function is an area of considerable interest (Kanai and Rees, 2011), but little is known about the specific brain basis of the reality monitoring variability in healthy individuals. An obvious place to look is for anatomical structures in the vicinity of anteromedial PFC that exhibit morphological variation in the normal population, such as the paracingulate sulcus (PCS). Located dorsal and parallel to the cingulate sulcus, the PCS is among the last sulci to develop in utero, and for this reason it varies greatly in size between individuals (Vogt et al., 1995; Paus et al., 1996). A prominent PCS can be observed in some healthy volunteers using structural MRI, whereas in others the PCS can appear to be absent (Fig. 2). Its presence can have a dramatic effect on the cytoarchitecture, gray matter volume, and thickness of surrounding cortex, with possible implications for cognition (Vogt et al., 1995; Fornito et al., 2008).

Specific PCS reductions have been observed in schizophrenia (Noga et al., 1995; Fornito et al., 2006b) with one study, for example, reporting complete absence bilaterally in $44 \%$ of schizophrenia patients, compared with $27 \%$ of controls (Yücel et al., 2002b). As schizophrenia is associated with impaired reality monitoring (Keefe et al., 2002), PCS reductions may have func- 


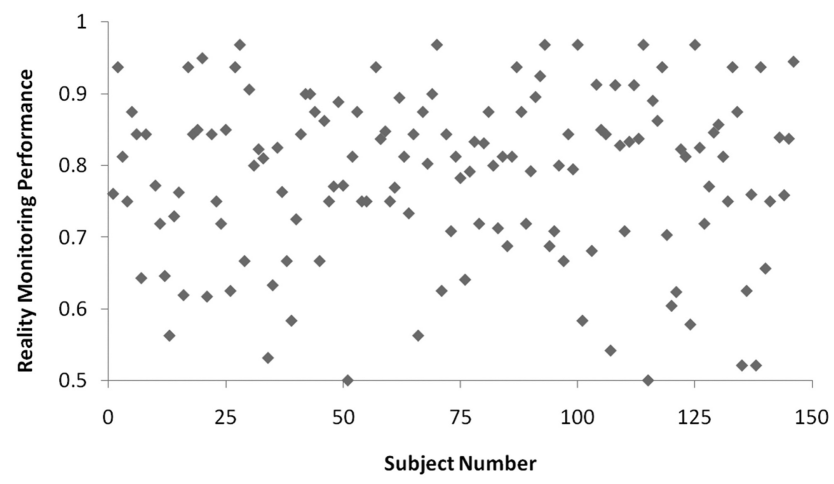

Figure 1. Performance of 146 healthy young adult volunteers who had undertaken one of a number of different reality monitoring tasks, illustrating the substantial individual differences in performance even in such a young, healthy sample. All tasks were two-alternative forcedchoice, so chance performance would be 0.5 .

tional significance for discriminating internally generated from externally generated information. Here, we investigate whether PCS structural variability can be used to explain the individual differences in reality monitoring observed in healthy individuals by using both visual classification and data-driven, voxel-based morphometry (VBM) methods. On the basis of the schizophrenia findings described above, we predicted that PCS absence in both hemispheres would be reflected in relatively poorer reality monitoring performance.

\section{Materials and Methods}

Participants. Fifty-three participants (23 males, 30 females) were recruited from a pool of healthy volunteers who had received an MRI scan as part of ongoing research projects at the Medical Research Council Cognition and Brain Sciences Unit, Cambridge. Participants were selected for recruitment on the basis of structural MRI scan classification of the PCS identifying clear presence or absence in the left or right hemisphere. The mean age was 26.9 years $(\mathrm{SD}=9.25$ years), and participants had a mean of 16.8 years of education ( $S D=2.65$ years). After a complete description of the study, written informed consent was obtained in a manner approved by the University of Cambridge Psychology Research Ethics Committee.

Reality monitoring task. The computer-based reality monitoring memory task was adapted from that used by Simons et al. (2006, 2008). The stimuli consisted of 160 well known word pairs (e.g., "Jekyll and Hyde," "Yin and Yang"). There were five blocks in total, each block containing a study and test phase. In the study phase, the first word to appear at the top of the screen was "SUBJECT" or "EXPERIMENTER," indicating who was to read out loud the word pair that would subsequently be presented in the center of the screen. After $500 \mathrm{~ms}$, either a word pair (Jekyll and Hyde: perceive condition) or the first word in the word pair and a question mark (Jekyll and ?: imagine condition) were shown. In the perceive condition, the subject or the experimenter read the word pair aloud. In the imagine condition, the subject or experimenter imagined the word that completed the pair and vocalized the whole word pair. Stimuli assignments to subject/experimenter and perceive/imagine conditions were counterbalanced between subjects, and the trial order was pseudorandomized such that no more than three consecutive trials were of the same condition.

Immediately following each study phase, participants' reality monitoring ability was assessed in a test phase. To test participants' recollection of whether they themselves or another person had read each word pair aloud, the following cue was presented: "Did either you or the experimenter read this word pair aloud?" Participants were then shown the first word of the word pair (e.g., Jekyll) in the center of the display with a reminder instruction underneath, and were instructed to press " 1 " on the keyboard if they thought they had read the pair in the study phase, and " 2 " if they believed the experimenter had read it aloud. To test partici- pants' ability to discriminate between perceived and imagined information, they were first shown the cue: "Was the accompanying word either perceived or imagined?" and were told to press " 1 " if they believed that the second word had been presented, or " 2 " if they thought that the accompanying word had been imagined. For each response, participants indicated how confident they were by holding down the response key as a confidence bar at the bottom of the screen increased in size. Participants had $4.5 \mathrm{~s}$ to start making their response. The type of recollection cued for each item and the ordering of test conditions were systematically counterbalanced between subjects.

Classification of sulcal variability. T1-weighted spoiled gradient echo structural MRI scans were obtained using a $3 \mathrm{~T}$ Siemens Tim Trio system. The PCS classifications were undertaken based on a protocol devised by Yücel et al. (2001) using freely available software (http://surfer.nmr.mgh.harvard.edu). An individual's PCS was identified as "prominent" if there was a salient horizontal sulcus running dorsal and parallel to the cingulate sulcus for at least $40 \mathrm{~mm}$, and was observable for at least three contiguous sagittal slices. A PCS was classified as "absent" if there was no indication of a PCS in the relevant slices (for examples, see Fig. 2). Each PCS was classified by two independent raters (M.B. and A.F.), with any disagreements resolved by discussion. Subjects with either a prominent or absent PCS in each hemisphere were selected for recruitment to the current study. This resulted in the following four PCS groupings: prominent in the left and right hemispheres ( $\mathrm{p}-\mathrm{p} ; n=16)$; prominent leftabsent right ( $\mathrm{p}-\mathrm{a} ; n=11)$; absent left-prominent right (a-p; $n=13)$; and absent in both hemispheres ( $\mathrm{a}-\mathrm{a} ; n=13)$. The groups did not differ significantly in terms of age $\left(F_{(3,52)}=1.42, p=0.25\right), \operatorname{sex}\left(F_{(3,52)}=0.29, p=0.83\right)$, or years of education $\left(F_{(3,52)}=0.37, p=0.78\right)$. Participants were selected for inclusion without any knowledge of their cognitive profile, other than that there was no history of neurological or psychiatric disorder.

VBM analysis. For the VBM analysis, structural MRI scans for each participant were preprocessed in SPM5 (Wellcome Trust Centre for Neuroimaging, London, UK) using a procedure based on the optimized VBM protocol (Good et al., 2001). All images were manually reoriented and spatially normalized into Montreal Neurological Institute (MNI) stereotactic space (Cocosco et al., 1997) before being segmented into gray matter, white matter, and CSF components. The four PCS groups did not differ in total intracranial volume $\left(F_{(3,52)}=0.08, p=0.97\right)$, or in volume of any of the segmented components (all $F$ values $<1$ ). The segmented images were spatially smoothed with an $8 \mathrm{~mm}$ full-width half-maximum isotropic Gaussian kernel. Two a priori regions of interest were defined as $10 \mathrm{~mm}$ spheres centered on medial anterior PFC coordinates from the two previous $\mathrm{fMRI}$ studies that involved our reality monitoring task (Simons et al., 2006, 2008). Multiple regression was used to identify clusters in which gray matter density correlated with reality monitoring performance at a threshold of $p<0.05$, small volume corrected (svc) for multiple comparisons within the regions of interest. Clusters outside the regions of interest were reported if they exceeded an uncorrected (unc) threshold of $p<0.001$. Brain regions and Brodmann areas (BA) were estimated from the Talairach and Tournoux (1988) atlas, after coordinates had been transformed between atlas spaces.

\section{Results}

Because our a priori prediction was that reduced reality monitoring would be seen in individuals with bilateral PCS absence, performance of participants in the a-a group was compared statistically with other participants using independent-samples $t$ tests (Stuss et al., 2005). Effect sizes were computed using Cohen's $d$ (Cohen, 1988). Reality monitoring accuracy is displayed in Figure 3, from which it can be seen that, as predicted, participants in the a-a group performed significantly worse overall than those in the other groups $\left(t_{(52)}=2.03, p<0.05, d=0.65\right)$. Performance broken down by condition (Table 1 ) indicated that this effect was significant for recollection of self/experimenter status $\left(\mathrm{t}_{(52)}=2.08, p<0.05, d=0.66\right)$, but not for perceived/ imagined recollection $\left(t_{(52)}=1.56, p=0.125, d=0.5\right)$, although there was no interaction $\left(F_{(1,51)}=0.11, p=0.75\right)$. The reality 
monitoring accuracy reduction occurred despite no significant differences between the a-a group and other participants in reaction times, either across all trials or focusing just on correct responses (all $t_{(52)}$ $<1, d<0.13$ ).

There was no between-group difference in trial-by-trial rated confidence, as indicated by participants' response duration measure. This suggests that those individuals in the a-a group may not have been aware of their significantly reduced reality monitoring ability. To confirm this, a metacognitive awareness score was computed for each participant by calculating the difference in mean rated confidence for correct and incorrect trials as a proportion of each participant's overall rated confidence level. This procedure produced a score between -1 and +1 , with higher positive scores indicating a good correspondence between confidence and accuracy. Consistent with the idea that participants in the a-a group lacked introspective awareness of their reality monitoring deficit, their metacognition scores (mean $=0.18, \mathrm{SD}=0.14$ ) were significantly reduced compared with those in the other groups ( $\mathrm{p}-\mathrm{p}$ : mean $=0.23, \mathrm{SD}=0.1 ; \mathrm{p}-\mathrm{a}$ : mean $=0.33, \mathrm{SD}=0.14$; a-p: mean $\left.=0.26, \mathrm{SD}=0.14 ; t_{(52)}=2.18, p<0.05, d=0.70\right)$.

As unequal group sizes can lead to violations of assumptions of the general linear model and thus increase statistical error, the observed a-a group differences were also assessed using a betweensubjects Monte Carlo permutation test, which is more robust against group size differences (Mewhort et al., 2009). Participant scores were randomly permuted without replacement, and the mean difference between the smaller and larger groups was calculated. This procedure was repeated 100,000 times, producing a random distribution of differences against which to test the significance of the observed mean difference. The number of times the permuted mean differences exceeded the observed mean difference was computed, and this value was divided by the total number of permutation samples, giving confirmed probabilities of the observed reality monitoring and metacognition score differences of $p<0.05$ at a two-tailed level.

Although participants were selected for inclusion on the basis of their PCS classification, it is possible that the observed behavioral effects might be attributable at least as much to structural differences elsewhere in the brain. To address this issue, VBM was used to identify any brain areas in which gray matter correlated significantly with reality monitoring performance across participants. First, we examined whether VBM was sufficiently sensitive to elicit an effect in the vicinity of the PCS. Because the predicted between-group effect described above was a reduction in reality monitoring performance if the PCS was absent, we reasoned that less sulcus might equate to greater surrounding cortical gray matter (Fornito et al., 2006a) and that we would observe a negative correlation between gray matter and reality monitoring.

As displayed in Figure 4, VBM regression analysis did indeed reveal a significant negative relationship where greater gray matter in the anterior PFC region of interest was associated with lower performance on the self/experimenter reality monitoring task $\left(x=22, y=58, z=2\right.$; BA $\left.10 ; Z=3.43 ; p<0.05_{\text {svc }}\right)$. Outside the region of interest, the only other areas to exhibit significant negative correlations with reality monitoring performance at a lower threshold of $p<0.001_{\text {unc }}$ were in the motor cortex. Positive correlations between gray matter and performance (at $p<$

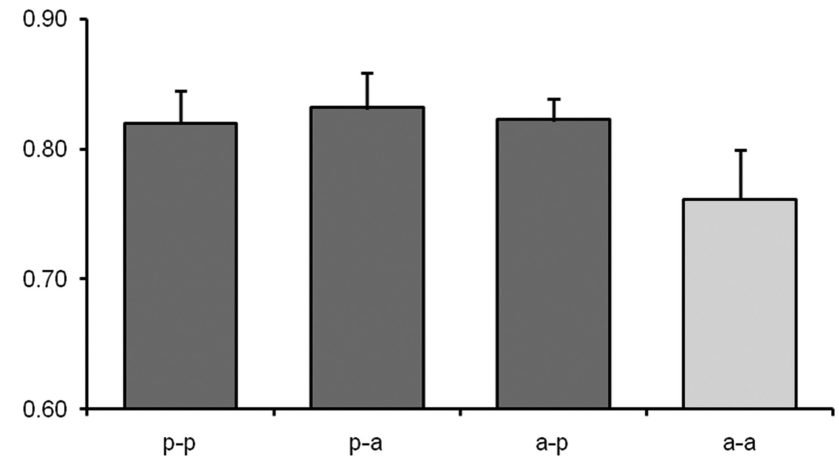

Figure 3. Reality monitoring performance of the four PCS groups.

Table 1. Accuracy and reaction time (ms) data

\begin{tabular}{llllll}
\hline & Accuracy & & & \multicolumn{2}{l}{ Reaction time } \\
Group & P/I & S/E & & P/I & S/E \\
\hline p-p & $0.77(0.15)$ & $0.87(0.07)$ & & $1786(240)$ & $1505(189)$ \\
p-a & $0.78(0.15)$ & $0.89(0.05)$ & & $1733(317)$ & $1558(227)$ \\
a-p & $0.78(0.09)$ & $0.86(0.05)$ & & $1974(243)$ & $1648(228)$ \\
a-d & $0.71(0.17)$ & $0.82(0.14)$ & & $1867(261)$ & $1583(270)$ \\
\hline
\end{tabular}

Data are given as mean (SD). P/I, Perceived/imagined; S/E, self/experimenter.

$\left.0.001_{\text {unc }}\right)$ were observed in areas including right parahippocampal cortex, the dorsal striatum, and precuneus (Table 2).

\section{Discussion}

The principal finding of the present experiment was that there may be a distinct neuroanatomical basis for the individual differences in reality monitoring ability typically seen in the healthy population. Volunteers in the a-a group, whose structural MRI scans indicated an absence bilaterally of the PCS, a tertiary sulcus in the medial anterior PFC, demonstrated significantly reduced reality monitoring ability compared with other participants. Moreover, the individuals in the a-a group appeared to lack introspective awareness of their reality monitoring deficit, scoring significantly lower in their metacognitive ability to discriminate correct from incorrect reality monitoring decisions, compared with those in the other PCS groups. Consistent with the prediction that PCS absence might mean greater volume in the surrounding frontal gyri, a data-driven, whole-brain analysis using VBM revealed significantly greater anterior PFC gray matter in individuals who performed poorly on the reality monitoring task. It is important to note that all participants in this experiment were healthy adult volunteers with typical educational backgrounds and no reported history of cognitive dysfunction due to neurological or psychiatric disorder. In this context, the observed 


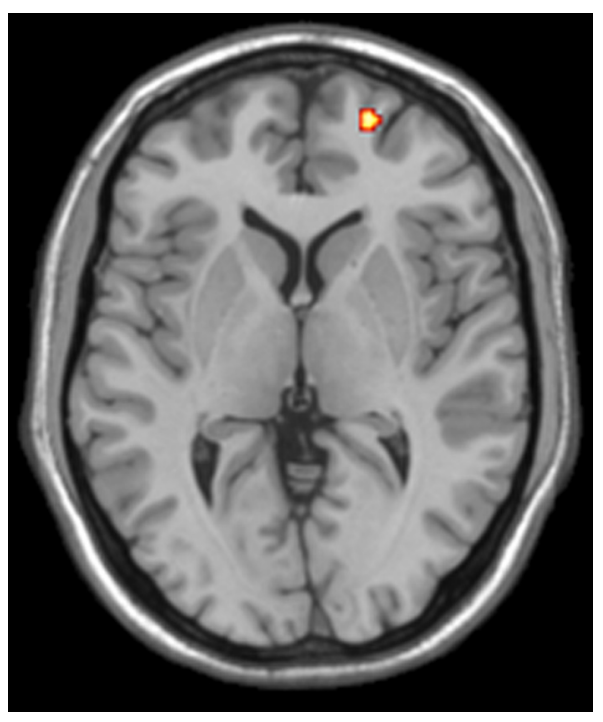

Figure 4. VBM analysis illustrating a significant correlation between greater anterior PFC gray matter and lower reality monitoring performance.

Table 2. Regions exhibiting significant correlations between gray matter volume and reality monitoring performance

\begin{tabular}{lrrrr}
\hline & \multicolumn{3}{l}{ Coordinates } & \\
\cline { 2 - 4 } Brain region & $x$ & $y$ & $z$ & $Z$ \\
\hline Negative correlations & 22 & 58 & 2 & 3.4 \\
$\quad$ Right anterior PFC (BA 10) & 26 & 8 & 64 & 3.6 \\
Right motor cortex (BA 6) & 32 & -6 & 64 & 3.7 \\
$\quad$ Right motor cortex (BA 6) & & & & \\
Positive correlations & -42 & 16 & 12 & 3.6 \\
$\quad$ Left ventrolateral PFC (BA 44) & 28 & 14 & 0 & 3.7 \\
Right putamen (BA 24) & -24 & 10 & 8 & 3.6 \\
Left putamen (BA 24) & 28 & -44 & -8 & 3.6 \\
Right parahippocampal gyrus (BA 36) & 54 & -68 & 12 & 3.4 \\
Right temporal cortex (BA 39) & 12 & -76 & -20 & 4.2 \\
Right occipital cortex (BA 18) & 14 & -78 & 46 & 3.5 \\
Right precuneus (BA 7) & & & & \\
\hline
\end{tabular}

Coordinates are in MNl atlas space (Cocosco et al., 1997).

differences in performance and structural brain volume exhibited by the a-a group are particularly striking.

The identification of a neuroanatomical basis for reality monitoring in medial anterior PFC is consistent with much recent functional neuroimaging evidence. Activity in this area is differentially modulated when contrasting recollection of which encoding task was previously undertaken with stimuli against remembering where on the screen (Simons et al., 2005b; Gilbert et al., 2010) or when in time (Simons et al., 2005a) the stimuli were presented, or remembering their size on the screen (Dobbins and Wagner, 2005). Focusing more closely on the kinds of reality monitoring tasks used in the present experiment, the same $\mathrm{PFC}$ region is involved in remembering whether verbal phrases were previously presented in full on the screen (e.g., "bacon and eggs"), or whether a word was missing that participants had to imagine (e.g., "bacon and ?") to complete the phrase (Simons et al., 2006; Vinogradov et al., 2006; Turner et al., 2008). Likewise, activity in this area is seen when the task is to differentiate whether oneself or the experimenter previously read a word pair aloud (Simons et al., 2008; Lagioia et al., 2011).

In addition to exhibiting a reality monitoring deficit, individuals in the a-a group were significantly impaired in their metamemory, the introspective ability to make judgments about their memory accuracy. Thus, their rated confidence in correct versus incorrect reality monitoring decisions was considerably less distinct than in the other participants. A number of previous neuroimaging studies have linked metamemory processes with regions of medial anterior PFC. For example, Schnyer et al. (2005) used an episodic feeling-of-knowing (FOK) paradigm to elicit predictions from participants about their likelihood of successfully retrieving sought-after information. Activity in this area was observed during accurate retrieval predictions, regardless of actual retrieval success. Maril et al. (2005) compared FOK judgments with another intermediate retrieval state, tip-of-thetongue, finding activity in a similar region that was sensitive to that kind of metacognitive judgment. Similarly, Chua et al. (2009) showed that the role for medial anterior PFC in metamemory generalized to making subjective confidence judgments about one's memory. Together, these findings are consistent with the proposal that the region plays a role in the introspective evaluation of stored memory contents (Moscovitch and Winocur, 2002).

Considering medial anterior PFC structures more closely, the present findings indicate that the PCS may have a specific deterministic role in supporting reality monitoring ability. Even though this is the first study to associate the PCS with reality monitoring, such a link is consistent with a number of converging lines of previous research. First, the medial PFC as a whole, and the region surrounding the paracingulate cortex in particular, exhibits considerable interindividual variability in sulcal and gyral anatomy. The PCS, which, when present, forms the superior and dorsal border to the paracingulate gyrus, is evident in $30-$ $60 \%$ of the normal population, most frequently in the left hemisphere (Paus et al., 1996). Functional consequences of this variability have been observed in performance on tests of executive function and cognitive control, such as spatial working memory and verbal fluency (Fornito et al., 2004). Second, PCS reductions have been reported in schizophrenia (Noga et al., 1995; Yücel et al., 2002b; Fornito et al., 2006b), a disorder in which patients often exhibit impaired reality monitoring (Keefe et al., 2002). Functional imaging studies of schizophrenia have associated the reality monitoring deficit seen in the disorder with reduced activity in the medial anterior PFC (Vinogradov et al., 2008). The specific relationship between PCS reductions in schizophrenia and reality monitoring impairment has yet to be investigated, although previous research has linked PCS variability in the disorder with performance variations on working memory tasks (Fornito et al., 2006b).

The observed association between PCS variability and reality monitoring suggests that such structural variability may directly influence the functional capacity of the medial PFC, which is consistent with evidence that PCS cortical folding variations can affect local activation patterns (Crosson et al., 1999; Yücel et al., 2002a; Amiez et al., 2006). The correlation between anterior PFC gray matter and reality monitoring performance in our data suggests that this influence is mediated through the effects of PCS variability on the morphometry (e.g., gray matter volume, thickness, surface area) of surrounding cortex, consistent with past work demonstrating that the morphometric consequences of such variations are directly correlated with cognitive abilities (Fornito et al., 2008). Such changes may reflect alterations in neuronal or synaptic density, or differences in the degree of intraregional versus interregional connectivity, given that cortical folds are thought to emerge as a product of gradients in the tension of axons linking proximal and distal areas (Van Essen, 1997). The precise relationship between variations in cortical folding 
and connectional anatomy are unclear, but it could be further explored using diffusion-based imaging.

This is the first study we are aware of that has investigated the structural basis of individual differences in reality monitoring in healthy individuals. However, a recent study used VBM to examine gray matter differences underlying performance variability in a nonmemory task that might require the kind of metacognitive awareness needed for reality monitoring. Fleming et al. (2010) asked participants to perform a simple perceptual decision task and rate their confidence in each decision that they made. Participants' ability to accurately link their confidence with their successful performance on the task was found to correlate positively with gray matter volume in anterior PFC. Consistent with this anatomical characterization, VBM analysis in the present experiment identified a closely located region of anterior PFC in which gray matter correlated significantly with lower realitymonitoring performance.

The negative correlation observed in the present data fits with the finding of the PCS analysis that sulcal absence, and thus, perhaps, greater volume of the surrounding gray matter (Fornito et al., 2006a), was associated with reality monitoring impairment. Similar negative relationships between regional brain volume and performance have been reported in a number of previous studies (Hyde et al., 2007; Dumontheil et al., 2010; Kanai et al., 2011), including one that linked the ability to manipulate selfgenerated thoughts in adolescence with reduced anterior PFC gray matter density (Dumontheil et al., 2010). It should be noted, however, that participants in the present study were specifically selected because MRI scans indicated particularly clear PCS presence or absence; it remains to be seen whether a similar negative correlation is observed in a random sample of the normal population. The VBM analysis in the present experiment also identified positive correlations between gray matter and performance in regions that included right parahippocampal cortex, the dorsal striatum, and precuneus, all areas known for their roles in learning and memory (Poldrack and Packard, 2003; Cavanna and Trimble, 2006). The present findings suggest that future VBM studies, and, for that matter, functional neuroimaging studies, should take account of whether their results could be influenced by structural morphological variations that may be present in participants.

In conclusion, the present study builds on previous work that has linked brain structure variability with individual differences in cognition, providing evidence that the variability in introspective abilities like reality monitoring typically seen in the healthy population may have a specific structural basis in the PCS. Because the defining morphological characteristics of the PCS are primarily determined in utero (Chi et al., 1977; Armstrong et al., 1995), one speculation is that the observed differences reflect a constraint imposed by early neurodevelopmental processes on the subsequent emergence of reality monitoring. As with all correlational data, it is not possible to establish a causal direction from the present findings, but it can be surmised with some certainty that individual differences in reality monitoring appear to have a specific structural basis in medial anterior PFC.

\section{References}

Amiez C, Kostopoulos P, Champod AS, Petrides M (2006) Local morphology predicts functional organization of the dorsal premotor region in the human brain. J Neurosci 26:2724-2731.

Armstrong E, Schleicher A, Omran H, Curtis M, Zilles K (1995) The ontogeny of human gyrification. Cereb Cortex 5:56-63.

Cavanna AE, Trimble MR (2006) The precuneus: a review of its functional anatomy and behavioural correlates. Brain 129:564-583.
Chi JG, Dooling EC, Gilles FH (1977) Gyral development of the human brain. Ann Neurol 1:86-93.

Chua EF, Schacter DL, Sperling RA (2009) Neural correlates of metamemory: a comparison of feeling-of-knowing and retrospective confidence judgments. J Cogn Neurosci 21:1751-1765.

Cocosco CA, Kollokian V, Kwan RKS, Evans AC (1997) Brainweb: online interface to a 3D MRI simulated brain database. Neuroimage 5:425.

Cohen J (1988) Statistical power analysis for the behavioral sciences, Ed 2. Hillsdale, NJ: LEA.

Crosson B, Sadek JR, Bobholz JA, Gökçay D, Mohr CM, Leonard CM, Maron L, Auerbach EJ, Browd SR, Freeman AJ, Briggs RW (1999) Activity in the paracingulate and cingulate sulci during word generation: an fMRI study of functional anatomy. Cereb Cortex 9:307-316.

Dobbins IG, Wagner AD (2005) Domain-general and domain-sensitive prefrontal mechanisms for recollecting events and detecting novelty. Cereb Cortex 15:1768-1778.

Dumontheil I, Hassan B, Gilbert SJ, Blakemore SJ (2010) Development of the selection and manipulation of self-generated thoughts in adolescence. J Neurosci 30:7664-7671.

Fleming SM, Weil RS, Nagy Z, Dolan RJ, Rees G (2010) Relating introspective accuracy to individual differences in brain structure. Science 329:1541-1543.

Fornito A, Yücel M, Wood S, Stuart GW, Buchanan JA, Proffitt T, Anderson V, Velakoulis D, Pantelis C (2004) Individual differences in anterior cingulate/paracingulate morphology are related to executive functions in healthy males. Cereb Cortex 14:424-431.

Fornito A, Whittle S, Wood SJ, Velakoulis D, Pantelis C, Yücel M (2006a) The influence of sulcal variability on morphometry of the human anterior cingulate and paracingulate cortex. Neuroimage 33:843-854.

Fornito A, Yücel M, Wood SJ, Proffitt T, McGorry PD, Velakoulis D, Pantelis C (2006b) Morphology of the paracingulate sulcus and executive cognition in schizophrenia. Schizophr Res 88:192-197.

Fornito A, Wood SJ, Whittle S, Fuller J, Adamson C, Saling MM, Velakoulis D, Pantelis C, Yücel M (2008) Variability of the paracingulate sulcus and morphometry of the medial frontal cortex: associations with cortical thickness, surface area, volume, and sulcal depth. Hum Brain Mapp 29:222-236.

Gilbert SJ, Henson RN, Simons JS (2010) The scale of functional specialization within human prefrontal cortex. J Neurosci 30:1233-1237.

Good CD, Johnsrude IS, Ashburner J, Henson RN, Friston KJ, Frackowiak RS (2001) A voxel-based morphometric study of ageing in 465 normal adult human brains. Neuroimage 14:21-36.

Hyde KL, Lerch JP, Zatorre RJ, Griffiths TD, Evans AC, Peretz I (2007) Cortical thickness in congenital amusia: when less is better than more. J Neurosci 27:13028-13032.

Hyman IE Jr, Billings FJ (1998) Individual differences and the creation of false childhood memories. Memory 6:1-20.

Johnson MK, Raye CL (1981) Reality monitoring. Psychol Rev 88:67-85.

Kanai R, Dong MY, Bahrami B, Rees G (2011) Distractibility in daily life is reflected in the structure and function of human parietal cortex. J Neurosci 31:6620-6626.

Kanai R, Rees G (2011) The structural basis of inter-individual differences in human behaviour and cognition. Nat Rev Neurosci 12:231-242.

Keefe RS, Arnold MC, Bayen UJ, McEvoy JP, Wilson WH (2002) Sourcemonitoring deficits for self-generated stimuli in schizophrenia: multinomial modeling of data from three sources. Schizophr Res 57:51-67.

Kensinger EA, Schacter DL (2006) Neural processes underlying memory attribution on a reality-monitoring task. Cereb Cortex 16:1126-1133.

Lagioia A, Eliez S, Schneider M, Simons JS, Van der Linden M, Debbané M (2011) Neural correlates of reality monitoring during adolescence. Neuroimage 55:1393-1400.

Maril A, Simons JS, Weaver JJ, Schacter DL (2005) Graded recall success: an event-related fMRI comparison of tip of the tongue and feeling of knowing. Neuroimage 24:1130-1138.

Mewhort DJ, Kelly M, Johns BT (2009) Randomization tests and the unequal-N/unequal-variance problem. Behav Res Methods 41:664-667.

Mitchell KJ, Johnson MK (2009) Source monitoring 15 years later: what have we learned from fMRI about the neural mechanisms of source memory? Psychol Bull 135:638-677.

Moscovitch M, Winocur G (2002) The frontal cortex and working with memory. In: Principles of frontal lobe function (Stuss DT, Knight RT, eds), pp 188-209. New York: Oxford UP. 
Noga JT, Aylward E, Barta PE, Pearlson GD (1995) Cingulate gyrus in schizophrenic patients and normal volunteers. Psychiatry Res 61:201-208.

Paus T, Tomaiuolo F, Otaky N, MacDonald D, Petrides M, Atlas J, Morris R, Evans AC (1996) Human cingulate and paracingulate sulci: pattern, variability, asymmetry, and probabilistic map. Cereb Cortex 6:207-214.

Poldrack RA, Packard MG (2003) Competition among multiple memory systems: converging evidence from animal and human brain studies. Neuropsychologia 41:245-251.

Schnyer DM, Nicholls L, Verfaellie M (2005) The role of VMPC in metamemorial judgments of content retrievability. J Cogn Neurosci 17:832-846.

Simons JS, Davis SW, Gilbert SJ, Frith CD, Burgess PW (2006) Discriminating imagined from perceived information engages brain areas implicated in schizophrenia. Neuroimage 32:696-703.

Simons JS, Gilbert SJ, Owen AM, Fletcher PC, Burgess PW (2005a) Distinct roles for lateral and medial anterior prefrontal cortex in contextual recollection. J Neurophysiol 94:813-820.

Simons JS, Owen AM, Fletcher PC, Burgess PW (2005b) Anterior prefrontal cortex and the recollection of contextual information. Neuropsychologia 43:1774-1783.

Simons JS, Henson RN, Gilbert SJ, Fletcher PC (2008) Separable forms of reality monitoring supported by anterior prefrontal cortex. J Cogn Neurosci 20:447-457.

Stuss DT, Alexander MP, Shallice T, Picton TW, Binns MA, Macdonald R, Borowiec A, Katz DI (2005) Multiple frontal systems controlling response speed. Neuropsychologia 43:396-417.

Talairach J, Tournoux P (1988) Co-planar stereotaxic atlas of the human brain. Stuttgart, Germany: Thieme.
Turner MS, Simons JS, Gilbert SJ, Frith CD, Burgess PW (2008) Distinct roles for lateral and medial rostral prefrontal cortex in source monitoring of perceived and imagined events. Neuropsychologia 46:1442-1453.

Van Essen DC (1997) A tension-based theory of morphogenesis and compact wiring in the central nervous system. Nature 385:313-318.

Vinogradov S, Luks TL, Schulman BJ, Simpson GV (2008) Deficit in a neural correlate of reality monitoring in schizophrenia patients. Cereb Cortex 18:2532-2539.

Vinogradov S, Luks TL, Simpson GV, Schulman BJ, Glenn S, Wong AE (2006) Brain activation patterns during memory of cognitive agency. Neuroimage 31:896-905.

Vogt BA, Nimchinsky EA, Vogt LJ, Hof PR (1995) Human cingulate cortex: surface features, flat maps, and cytoarchitecture. J Comp Neurol 359:490-506.

Yücel M, Pantelis C, Stuart GW, Wood SJ, Maruff P, Velakoulis D, Pipingas A, Crowe SF, Tochon-Danguy HJ, Egan GF (2002a) Anterior cingulate activation during stroop task performance: a PET to MRI coregistration study of individual patients with schizophrenia. Am J Psychiatry 159:251-254.

Yücel M, Stuart GW, Maruff P, Velakoulis D, Crowe SF, Savage G, Pantelis C (2001) Hemispheric and gender-related differences in the gross morphology of the anterior cingulate/paracingulate cortex in normal volunteers: an MRI morphometric study. Cereb Cortex 11:17-25.

Yücel M, Stuart GW, Maruff P, Wood SJ, Savage GR, Smith DJ, Crowe SF, Copolov DL, Velakoulis D, Pantelis C (2002b) Paracingulate morphologic differences in males with established schizophrenia: a magnetic resonance imaging morphometric study. Biol Psychiatry 52:15-23. 